Article

\title{
Impact of Co-Occurring Psychiatric Disorders on Retention in a Methadone Maintenance Program: An 18-Month Follow-Up Study
}

Mònica Astals ${ }^{1}$, Laura Díaz ${ }^{1}$, Antònia Domingo-Salvany ${ }^{2}$, Rocío Martín-Santos ${ }^{1}$, Antoni Bulbena ${ }^{1,3}$ and Marta Torrens ${ }^{1,3, *}$

1 Institute of Psychiatry and Addiction (IAPs), Hospital del Mar and Institut Municipal d'Investigació Mèdica (IMIM), Hospital del Mar, Psg. Marítim 25-29, E-08003 Barcelona, Spain;

E-Mails: mastals@imas.imim.es (M.A.); ldiaz@imim.es (L.D.); rmsantos@imim.es (R.M.S.); 16359@imas.imim.es (A.B.)

2 Research Programme in Epidemiology and Public Health, Institut Municipal d'Investigació Mèdica (IMIM), Hospital del Mar, Doctor Aiguader 88, E-08003 Barcelona, Spain;

E-Mail: adomingo@imim.es

3 Department of Psychiatry, Universitat Autònoma de Barcelona, Barcelona, Spain

* Author to whom correspondence should be addressed; E-Mail: mtorrens@imas.imim.es;

Tel.: +34-93-248-3175; Fax: +34-93-316-0410.

Received: 15 October 2009 / Accepted: 10 November 2009 / Published: 12 November 2009

\begin{abstract}
We assess the influence of co-occurring psychiatric disorders on retention in 189 opioid dependent patients in a methadone maintenance treatment (MMT) and determine the incidence of psychiatric co-morbidity during an 18-month follow-up period. About $68.5 \%$ were retained in the MMT. Neither co-occurring mental disorders (chi-square $=0.303$, $\mathrm{df}=1, \mathrm{p}=0.622$ ) nor methadone doses [85 (88.9) vs. 79.2 (85) $\mathrm{mg} / \mathrm{day}, \mathrm{p}=0.672]$ were related to retention. In the follow-up period 19 new diagnoses were made, mainly major depression and antisocial and borderline personality disorders. Co-occurring psychiatric disorders should be assessed during MMT follow-up.
\end{abstract}

Keywords: methadone; retention; co-occurring psychiatric disorders; incidence 


\section{Introduction}

In the management of heroin dependent subjects methadone maintenance treatment (MMT) programs are effective in reducing heroin use, crime related to drug use, HIV risk behaviors, overdose mortality [1-3] and improving quality of life [4,5]. Effectiveness is related to the effect of methadone on retaining patients in the program. Research into the factors related to retention in MMT is an important issue in order to improve the effectiveness of MMT.

Previous studies suggest that the patient must stay for at least one year in the MMT for the treatment to be effective [6,7] although longer treatment is recommended. In different studies, the 1-year retention rate ranges between $25 \%$ to $82 \%$ [6,8-13]. Factors associated with retention rate are methadone dosage [2,14], differences between treatment settings [15], severity of drug use at the time of enrolment in the program [16] and age at time of MMT entrance [17]. The influence of co-occurring mental disorders other than substance abuse has also been studied, although results remain controversial $[11,13,15,18]$. This could be mainly related to the difficulties in diagnosing co-occurring disorders in substance users [19,20] and to the fact that in previous studies the patients were only assessed at the time of admission into MMT and therefore the role of new non-substance use psychiatric diagnosis during the follow-up period was not considered.

A prospective follow-up study of opioid dependent patients included in the MMT [21] was carried out with the following objectives: (1) to assess the influence of co-occurring disorders on retention in the MMT program and (2) to determine the incidence of co-occurring disorders during an 18-month follow-up.

\section{Methods}

\subsection{Sample}

The study participants were 189 opioid dependent patients ( $77 \%$ male, mean age $34 \pm 7.5$ years) consecutively admitted to a MMT in Barcelona, Spain, and followed up to 18 months.

\subsection{Procedure}

In the baseline visit, after full explanation of the purposes of the study, written informed consent was obtained and patients were assessed with the PRISM-IV and a close-ended questionnaire (see other variables). At follow-up (18 months) patients were reassessed with the same measures. The study protocol was approved by the institutional review board.

\subsection{Methadone Maintenance Treatment}

The MMT provided at the Drug Abuse Out-patient Centre (CAS-BARCELONETA) is a low-threshold MMT, that is to say such program is not abstinence oriented. The only requirement for inclusion in the program is a definitive diagnosis of opioid dependence according to DSM-IV criteria. Forced discharge only occurs for aggressive behavior and drug trafficking in the centre. There is a high 
dose policy (no upper limit) and no restriction on long-term treatment (no time limit). The induction period on MMT lasts about 1-2 months until the stable maintenance doses is achieved. Urine toxicology screens are carried out randomly once a week under supervision. Methadone is dispensed to patients daily on the form of syrup with orange juice and has to be ingested in the presence of a nurse. Take-home methadone doses are provided when weekly urine screening tests are repeatedly clean (at least for a period of one month). Take-home privileges are revoked in response to positive urine tests results, and patients are referred to the clinicians to assess a possible increase in methadone doses. In addition to methadone and urine drug screen, individual counseling is the major therapeutic vehicle and frequency varies depending upon the stage of treatment and patient needs. Counseling focused on encouraging reduced drug use and helping patients to cope with problems (either through direct counseling or referral to other services) that made them more vulnerable to continued drug use is provided.

\subsection{Current Co-Occurring Diagnoses}

Diagnoses of current substance use disorder (SUD) and co-occurring mental disorders, were carried out according to DSM-IV criteria and using the Spanish version of the Psychiatric Research Interview for Substance and Mental Disorders (PRISM-IV) [22], administered by two trained psychologist researchers with clinical experience with patients with substance abuse or mental disorders. The PRISM-IV has shown a good test-retest reliability [23] and validity [22] in substance abusers.

Figure 1. Situation of the 189 opioid dependent patients admitted to a MMT at 18 months follow-up.

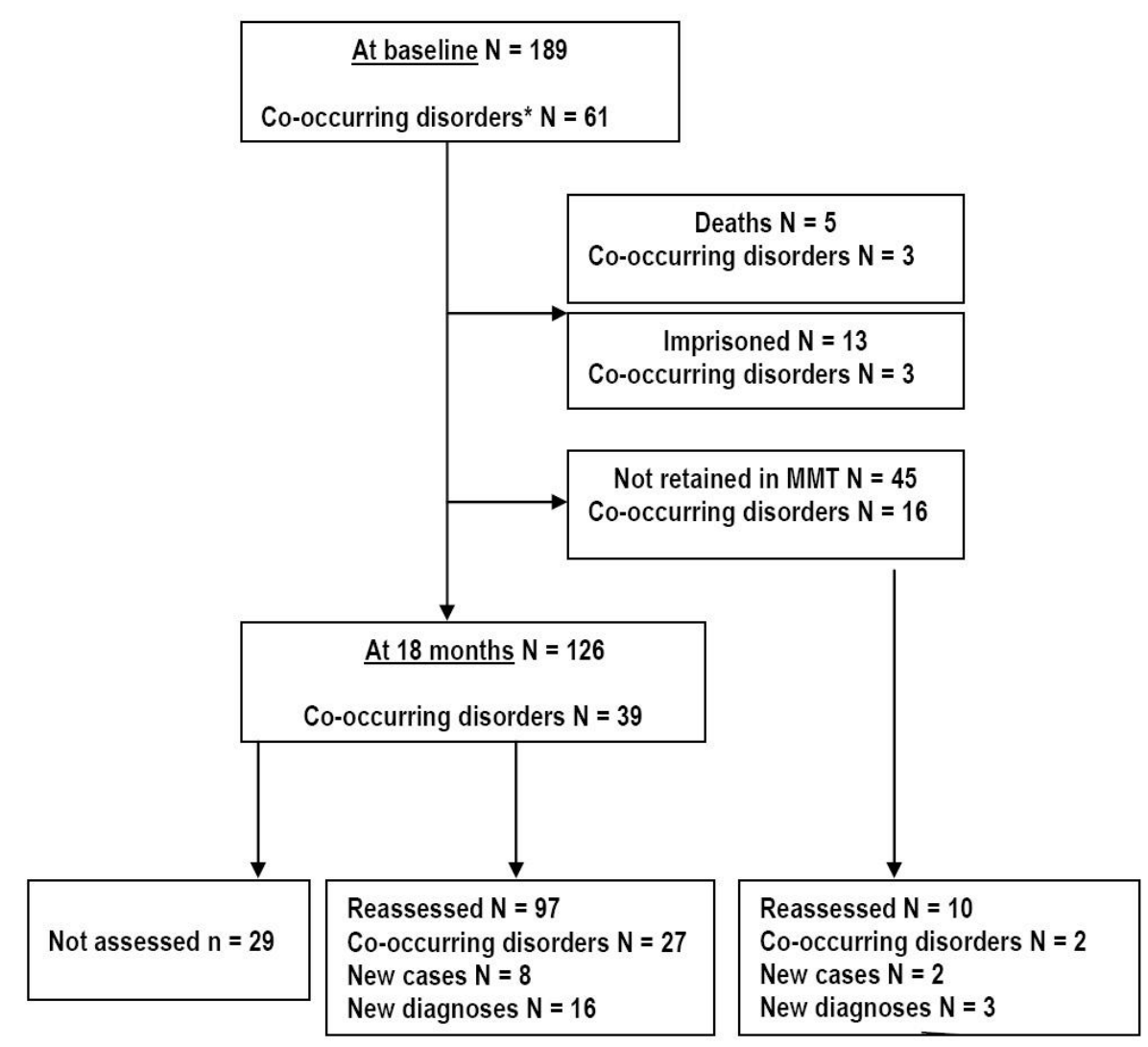

* Co-occurring disorders: co-occurring substance use [abuse or dependence] and mental disorders. 


\subsection{Other Variables}

Baseline patient's sociodemographic characteristics (including employment and legal status), and drug use and sexual risk behaviors, substance use variables, and infection by HIV and hepatitis $\mathrm{C}$ virus were collected with a close-ended questionnaire [21]. At follow-up, the questionnaire included data on MMT provided (methadone dose received at 18 months). In the patients not retained in the program, data on the last methadone dose administered before interruption of MMT was used.

\subsection{Statistical Analysis}

All statistical analyses were carried out with the SPSS statistical software package [SPSS V14; Chicago, IL, USA]. Retention in the MMT was defined as remaining in the same MMT program, and after stable doses of methadone was reached. The presence of co-occurring mental disorders at baseline between retained and non-retained patients was compared with Pearson's chi-square test. Fisher's exact test was used when one or more cells of the contingency tables had expected counts of less than five.

To determine which variables were associated with MMT retention, we compared retained versus non-retained patients in the following baseline variables: sociodemographic (sex, age, education level, employment, legal status); psychopathological (co-occurring mental disorders [yes/no]); use of the following substances during the last month: alcohol, sedatives, cocaine, cannabis, opiates other than heroine; SUD diagnoses (yes/no) of the following substances: alcohol, sedatives, cocaine, cannabis, opiates other than heroine; VIH/Hepatitis $\mathrm{C}$ risk-related variables: i.v. route use, sharing injection material, sexual risk behavior (yes/no for always using condoms); presence or absence of HIV-Ab and/or HCV-Ab; and methadone dose. Comparisons were made by means of Pearson's chi-square test or Fisher's exact test (for categorical variables) and Student's $t$-test (for continuous variables). A logistic regression analysis with retention as the dependent variable and those of the former variables that showed a $p$-value of less than 0.25 in the univariate analyses: sex, age, educational level, and current diagnosis of cocaine and opiates other than heroin dependence as independent variables was done. The goodness-of-fit of the model was assessed by the Hosmer-Lemeshow test.

To assess the incidence of co-occurring disorders during the follow-up, the cumulative incidence (ratio of incident cases divided by those subjects without co-occurring disorders at baseline) and the incidence rate (ratio of new diagnoses divided by total time of follow-up) of retained cases were calculated.

Exact $95 \%$ confidence intervals for the incidence rate based on the Poisson distribution were calculated.

\section{Results}

Table 1 shows a summary of the baseline characteristics of the sample differentiating among those $128(67.7 \%)$ cases with only a current SUD diagnosis and 61 (32.3\%) with a current co-occuring mental disorders. Anxiety disorders were the most frequent Axis-I diagnoses, followed by mood 
disorders, and psychotic disorders. More than $20 \%$ of the sample fulfilled the criteria for antisocial or borderline personality disorder.

Table 1. Differences between patients with/without co-occurring disorders at baseline.

\begin{tabular}{|c|c|c|c|c|c|}
\hline Variable & $\begin{array}{c}\text { Co-occurring } \\
\text { disorders } \\
\mathrm{N}=61(\%) \\
\end{array}$ & $\begin{array}{c}\text { Only SUD } \\
\mathrm{N}=128(\%)\end{array}$ & $\chi^{2}$ or $t$ & df & $p$ \\
\hline Women & $20(32.8)$ & $24(18.8)$ & $\chi^{2}=4.557$ & 1 & 0.043 \\
\hline Age, years, mean (SD) & $33.34(8.8)$ & $34.1(6.8)$ & $t=-0.637$ & 187 & 0.525 \\
\hline \multicolumn{6}{|l|}{ Marital status } \\
\hline Single & $42(68.9)$ & $60(46.9)$ & \multirow{3}{*}{$\chi^{2}=8.079$} & \multirow{3}{*}{2} & \multirow{3}{*}{0.018} \\
\hline Currently married & $12(19.7)$ & $45(35.2)$ & & & \\
\hline Separated/divorced & $7(11.5)$ & $23(18)$ & & & \\
\hline \multicolumn{6}{|l|}{ Education level } \\
\hline Primary School & $29(47.5)$ & $47(36.7)$ & \multirow{3}{*}{$\chi^{2}=2.018$} & \multirow{3}{*}{2} & \multirow{3}{*}{0.365} \\
\hline Secondary School & $20(32.8)$ & $50(39.1)$ & & & \\
\hline University & $12(19.7)$ & $31(24.2)$ & & & \\
\hline Any month employed last six months & $26(42.6)$ & $87(68)$ & $\chi^{2}=11.039$ & 1 & 0.001 \\
\hline \multicolumn{6}{|l|}{ Legal status } \\
\hline Any detention last six months & $20(32.8)$ & $24(18.8)$ & \multirow{2}{*}{$\begin{array}{l}\chi^{2}=4.557 \\
\chi^{2}=0.398\end{array}$} & 1 & 0.043 \\
\hline Any imprisonment last 6 months & $7(11.5)$ & $11(8.6)$ & & 1 & 0.598 \\
\hline \multicolumn{6}{|l|}{ Serological status } \\
\hline $\mathrm{HIV}$ - Ab positive & $18(29.5)$ & $27(21.8)$ & \multirow{2}{*}{$\begin{array}{l}\chi^{2}=1.329 \\
\chi^{2}=3.747\end{array}$} & 1 & \multirow{2}{*}{$\begin{array}{l}0.276 \\
0.059\end{array}$} \\
\hline $\mathrm{HCV}-\mathrm{Ab}$ positive & $39(63.9)$ & $58(48.7)$ & & 1 & \\
\hline \multicolumn{6}{|l|}{ HIV current risk behaviors last six } \\
\hline Any i.v. drug use & $30(49.2)$ & $73(57)$ & \multirow{2}{*}{$\begin{array}{l}\chi^{2}=5.215 \\
\chi^{2}=1.027\end{array}$} & 1 & \multirow{2}{*}{$\begin{array}{l}\mathbf{0 . 0 2 9} \\
0.350\end{array}$} \\
\hline \multirow{2}{*}{\multicolumn{6}{|c|}{ Any sexual risk behavior }} \\
\hline & & & & & \\
\hline Alcohol & $5(8.2)$ & $23(18)$ & \multirow{5}{*}{$\begin{array}{l}\chi^{2}=3.127 \\
\chi^{2}=2.402 \\
\chi^{2}=4.423 \\
\chi^{2}=0.084 \\
\chi^{2}=0.586\end{array}$} & 2 & 0.209 \\
\hline Cocaine & $10(16.4)$ & $15(11.7)$ & & 2 & 0.301 \\
\hline Sedatives & $15(24.6)$ & $16(12.5)$ & & 2 & 0.110 \\
\hline Other opiates & $9(14.8)$ & $21(16.4)$ & & 2 & 0.834 \\
\hline Cannabis & $18(29.5)$ & $35(27.3)$ & & 2 & 0.746 \\
\hline \multicolumn{6}{|l|}{ Cocaine route use } \\
\hline Snorted/Smoked & $13(38.2)$ & $27(50)$ & \multirow[t]{2}{*}{$\chi^{2}=1.165$} & \multirow[t]{2}{*}{1} & \multirow[t]{2}{*}{0.380} \\
\hline Injected & $21(61.8)$ & $27(50)$ & & & \\
\hline \multicolumn{6}{|l|}{ Heroine route use } \\
\hline Smoked/Inhaled & $12(18.7)$ & 37 (28.9) & \multirow{3}{*}{$\chi^{2}=4.770$} & \multirow{3}{*}{1} & \\
\hline Snorted & $11(18)$ & $33(25.8)$ & & & 0.092 \\
\hline Injected & $38(62.3)$ & $58(45.3)$ & & & \\
\hline Abuse or dependence diagnoses & & & & & \\
\hline Alcohol & $6(9.8)$ & $12(9.4)$ & $\chi^{2}=0.010$ & 1 & 1 \\
\hline Other opiates & $4(6.6)$ & $9(7)$ & $\chi^{2}=0.014$ & 1 & 1 \\
\hline Cocaine & $25(41)$ & $43(33.6)$ & $\chi^{2}=0.979$ & 1 & 0.335 \\
\hline Sedatives & $11(18)$ & $18(14.1)$ & $\chi^{2}=0.501$ & 1 & 0.520 \\
\hline Cannabis & $17(27.9)$ & $20(15.6)$ & $\chi^{2}=3.934$ & 1 & 0.053 \\
\hline Stimulants & $2(3.3)$ & $2(1.6)$ & $\chi^{2}=0.587$ & 1 & 0.596 \\
\hline
\end{tabular}


Table 1. Cont.

\begin{tabular}{lcc}
\hline Non-SUD co-occurring diagnoses & \\
Only Axis I & $32(52.5)$ & - \\
Only Axis II & $22(36.1)$ & - \\
Both Axis I + II & $7(11.5)$ & - \\
\hline Major depression & $9(14.8)$ & \\
Induced depression & $5(8.2)$ & \\
Schizophrenia & $5(8.2)$ & \\
Panic w/without agoraphobia & $12(19.7)$ & \\
Social phobia & $10(16.4)$ & \\
Simple phobia & $8(13.1)$ & \\
Post traumatic stress & - & \\
Obsessive compulsive & $4(6.6)$ & \\
Bulimia & $1(1.6)$ & \\
\hline Antisocial Personality Disorder & $16(26.2)$ \\
Borderline Personality Disorder & $13(21.3)$ \\
\hline
\end{tabular}

Co-occurring diagnoses: co-occurring substance use [abuse or dependence] and mental disorders; Only

SUD: Only substance use disorders.

The situation of patients during the 18-month follow-up period is shown in Figure 1. Sixty-three of the 189 patients dropped out the study because of death, imprisonment or MMT discontinuation. Therefore, a total of 126 patients were followed, with a retention rate of $68.5 \%$. Some baseline differences between the 126 patients who continued in the MMT and the 63 patients who dropped out were found. Subjects that dropped out used more cocaine, $(57.1 \%$ vs. 38.1\%; chi square $=7.510$, $\mathrm{df}=2, \mathrm{p}=0.023)$, reported more i.v. route $(65.1 \%$ vs. $42.9 \%$; chi square $=8.297, \mathrm{dfl}=1, \mathrm{p}=0.005)$ and less sexual risk behaviors $(36.5 \%$ vs. $63.5 \%$; chi square $=12.333, \mathrm{dfl}=1, \mathrm{p}=0.001)$ during last 30 days before starting in the MMT. Furthermore, subjects that dropped out had more past $(68.3 \%$ vs. $51.6 \%$; chi square $=4.764$, df $=1, \mathrm{p}=0.030)$ and current $(44.4 \%$ vs. $27 \%$; chi square $=5.809, \mathrm{df}=1, \mathrm{p}=0.021)$ cocaine dependence diagnoses than subjects who continued in the MMT.

Twenty-nine patients were not available for reassessment at follow-up, even if still in MMT. Baseline differences were found respect to those 97 patients retained in MMT and reassessed at follow up. The 29 who did not accepted to be reassessed used more the i.v. route $(65.5 \%$ vs. $36.1 \%$; chi square $=7.898, \mathrm{df}=1, \mathrm{p}=0.006)$ and higher alcohol $(17.2 \%$ vs. $13.4 \%$; chi square $=7.236, \mathrm{df}=2$, $\mathrm{p}=0.027)$ and cocaine $(58.6 \%$ vs. $19.6 \%$; chi square $=17.731 \mathrm{df}=2, \mathrm{p}<0.001)$ in the 30 days before entering MMT. Furthermore, the 29 patients had more antisocial personality disorder diagnoses $(17.2 \%$ vs. $4.1 \%$; chi square $=5.792, \mathrm{df}=1, \mathrm{p}<0.030)$ (data not shown).

Of the 61 patients with co-occurring mental disorders at baseline, $39(63.9 \%)$ were retained in the MMT programme and $22(36.1 \%)$ were lost to follow-up, whereas from the 128 without co-occurring mental disorders, $87(68 \%)$ patients were retained (chi-square $=0.303$, $\mathrm{df}=1, \mathrm{p}=0.622$ ). No difference in baseline characteristics were observed between the patients retained in MMT and available for the reassessment at time of follow-up $(n=97)$ and those retained but not available for the reassessment $(\mathrm{n}=29)$. The difference between methadone dosage of those patients retained in the MMT and last dose administered in those who dropped out was not statistically significant [85.5 (88.9) vs. 79.2 (85) mg/day, $\mathrm{p}=0.672$ ]. None of the variables included in the multivariate model 
were significant predictors of treatment retention, although being male (OR: $2.59,95 \%$ CI: $0.98-6.84$; $\mathrm{p}=0.055)$ and lower educational level (OR: 2.65, 96\% CI: 0.98-7.13; p = 0.054) were nearly significant predictors of non-retention. The p-value of the Hosmer-Lemeshow tests was 0.47 indicating that the model fit was satisfactory.

A total of 107 patients were available for the assessment of co-occurring mental disorders at follow-up. Co-occurring disorders were established in 29 subjects. When patients with $(\mathrm{n}=29)$ and without $(n=78)$ co-occurring disorders were compared, those with co-occurring disorders had worked fewer months before admission in the MMT $(41.4 \%$ vs. $69.2 \%, \mathrm{p}=0.013)$ and before reassessment at follow-up $(24.1 \%$ vs. $50 \%, \mathrm{p}=0.027)$, showed higher percentage of current alcohol abuse diagnoses $(13.8 \%$ vs. $1.3 \%, \mathrm{p}=0.019)$ and had received more psychiatric treatment $(44.8 \%$ vs. $19.2 \%$, $\mathrm{p}=0.012$ ).

In 10 of the 107 patients, at least one new co-occurring disorder was diagnosed, with a total of 19 diagnoses. Sixty percent of these 10 patients were men, with a mean age of 31.60 (5.73) years. The cumulative incidence of co-occurring disorders was $13 \%$ (95\% CI $6.4 \%$ to $22.5 \%$ ). The cumulative incidence in the 97 patients retained in the MMT program and reassessed was $11.43 \%$ (95\% CI 5.07\% to $21.28 \%$ ) compared with $28.6 \%$ (95\% CI $3.7 \%$ to $71 \%$ ) in 10 patients reassessed but not retained in the MMT program.

Of the 19 new diagnoses, 16 were made in the 97 patients retained in MMT and reassessed, and 3 in the subsample of 10 patients not retained but reassessed. The incidence rate of co-occurring disorders at follow-up was 0.11 diagnoses per year (95\% CI 0.06 to 0.18 ) in patients retained in MMT and 0.20 (95\% CI 0.04 to 0.58 ) in those not retained. Nine new Axis I diagnoses were done: major depression (five diagnoses), anxiety disorders (one panic disorder and one specific phobia) and psychotic disorder (one induced and one primary). Regarding Axis II disorders, 10 new diagnoses had been made of personality disorders (five antisocial personality disorder [APD] and five borderline personality disorder [BPD]); see Table 2 for more details.

Table 2. Co-occurring disorders incidence in a cohort of 107 patients reassessed at 18 months.

\begin{tabular}{|c|c|c|c|c|}
\hline \multirow{3}{*}{ Disorders at baseline } & \multicolumn{4}{|c|}{ Co-occurring disorders } \\
\hline & Baseline & & Follow-up & \\
\hline & & Axis I & Axis II & Axis I \& II \\
\hline Only SUD & 77 & $\begin{array}{l}1 \text { Induced } \\
\text { Psychosis }^{\mathrm{a}} \\
1 \text { Simple Phobia } \\
1 \text { Schizophrenia }\end{array}$ & $\begin{array}{l}1 \mathrm{APD} \& \mathrm{BPD}^{\mathrm{a}, \mathrm{b}} \\
1 \mathrm{APD} \\
2 \mathrm{BPD}\end{array}$ & \\
\hline Non SUD co-occurring diagnoses & 30 & & & \\
\hline Axis I & 19 & & & \\
\hline Major Depression & 7 & & $1 \mathrm{BPD}$ & $\begin{array}{l}1 \text { Induced } \\
\text { Depression \& } \\
\text { BPD }^{\mathrm{b}}\end{array}$ \\
\hline Induced Depression & 1 & & & \\
\hline Schizophrenia & 2 & & & \\
\hline Induced Psychosis & - & & & \\
\hline Panic w/without Agoraphobia & 9 & 3 Major Depress & & \\
\hline Social Phobia & 6 & & $2 \mathrm{APD}$ & \\
\hline
\end{tabular}


Table 2. Cont.

\begin{tabular}{lll}
\hline Simple Phobia & 4 & \\
\hline Obsessive Compulsive & 3 & \\
\hline Axis II & 8 & \\
\hline Antisocial Personality & 5 & \\
\hline Borderline Personality & 6 & \\
\hline Axis I \& II & 3 & \\
\hline $\begin{array}{l}\text { Social Phobia, Induced Depression } \\
\text { \& BPD }\end{array}$ & 1 & $\begin{array}{l}\text { 1 Major } \\
\text { Depression }\end{array}$ \\
\hline Panic with/without Agoraphobia \& & 1 & $\begin{array}{l}\text { 1 Major } \\
\text { Depression }\end{array}$ \\
BPD & & \\
\hline Panic with/without Agoraphobia \& & 1 & \\
APD & & \\
\hline
\end{tabular}

${ }^{a}$ Patients from the subsample of 10 patients not retained but assessed; ${ }^{\mathrm{b}}$ Both diagnoses in single patient;

APD: Antisocial personality disorder; BPD: Borderline personality disorder.

\section{Discussion}

In this study, the overall cumulative incidence of co-occurring disorders in methadone-treated opioid dependent patients followed-up for 18 months was 13\%. Major depression and antisocial and borderline personality disorders were the most common new diagnoses. The cumulative incidence, although not statistically significant, was lower in patients retained in MMT than those not retained. Moreover, co-occurring disorders at the time of inclusion in the MMT was not related to retention in treatment.

Major depression has been the main incident Axis I diagnoses. Interestingly, all those patients had been diagnosed with an anxiety disorder at baseline. Our results are similar to those obtained by others at 12 months follow-up after discharge of inpatient detoxification treatment using similar methodology [20], but differ from those of Grant et al. [24], suggesting that anxiety disorders at baseline more often predicted incidence of anxiety disorders rather than mood disorders in the general population. The establishment of new personality disorders was indeed quite surprising, since a personality disorder implies a pattern of behaviors developing through one's adult life, beginning in the early adulthood. Such result could be explained by the fact that at baseline patients were diagnosed just at admission of MMT and it was more difficult to differentiate personality than substance related symptoms. In agreement with other authors [13,25], we consider that the diagnoses of personality disorders should be done once the patients have become clinically stabilized.

Although in the univariate analysis, cocaine use as IV use at entrance, was a predictor of drop-out of treatment in agreement with previous studies[26], in the multivariate regression analysis, no significant relationship was proven between these variables and the retention in MMT. Nor did we find other significant predictor of retention in MMT, although statistical significance was almost reached for two variables (gender and education level). It seems that patients with a lower educational level might need some specific treatment approaches, such as mapping-enhanced counseling to improve treatment outcomes. Previous studies [27,28] found controversial results regarding education level and MMT retention. We did not observe a relationship between methadone dose and retention in treatment, probably because mean dose of methadone administered (near $80 \mathrm{mg} /$ day in both groups) was in the 
recommended range [14]. Psychiatric comorbidity did not show any influence on patient's retention in MMT, as previously described by other authors[11,13,18].

Some study limitations should be mentioned. Firstly, the lack of the assessment of psychosocial interventions received during the treatment, and secondly, the fact that co-occurring mental disorders were only evaluated in a small percentage of subjects not retained in the MMT.

In summary, the present study shows a relatively high cumulative incidence of co-occurring diagnoses (mainly major depression) among opioid dependent patients available at follow-up. Furthermore, the unexpected rate of new personality disorder diagnoses established in MMT retained patients enhances the relevance of careful detection of co-occurring mental disorders not only at the time admission to the treatment, but also throughout the whole program.

\section{Funding and Support}

Conflicts of interest: Not declared. This study was supported by grants from "Plan Nacional sobre Drogas" (2SI/01/07) and "Fondo de Investigación Sanitaria" (FIS) (G03/005 and PI052086), Madrid, Spain.

\section{Acknowledgements}

The authors are grateful to the nurses of the primary drug treatment centers (CAS Creu Roja, CAS Sants and CAS Barceloneta) for their participation in the recruitment of subjects, Klaus Langhor, MD, and Albert Sanchez-Niubó for their contribution in the statistical analysis, and Marta Pulido, MD, for editing the manuscript and editorial assistance.

\section{References}

1. Marsch, L.A. The efficacy of methadone maintenance interventions in reducing illicit opiate use, HIV risk behavior and criminality: a meta-analysis. Addiction 1998, 93, 515-532.

2. Amato, L.; Davoli, M.; Perucci, C.A.; Ferri, M.; Faggiano, F.; Mattick, R.P. An overview of systematic reviews of the effectiveness of opiate maintenance therapies: available evidence to inform clinical practice and research. J. Subst. Abuse Treat. 2005, 28, 321-329.

3. Brugal, M.T.; Domingo-Salvany, A.; Puig, R.; Barrio, G.; Garcia, D.O.; de la Fuente L. Evaluating the impact of methadone maintenance programmes on mortality due to overdose and aids in a cohort of heroin users in Spain. Addiction 2005, 100, 981-989.

4. Torrens, M.; San, L.; Martinez, A.; Castillo, C.; Domingo-Salvany, A.; Alonso, J. Use of the Nottingham Health Profile for measuring health status of patients in methadone maintenance treatment. Addiction 1997, 92, 707-716.

5. Torrens, M.; Domingo-Salvany, A.; Alonso, J.; Castillo, C.; San, L. Methadone and quality of life. Lancet 1999, 353, 1101.

6. Friedmann, P.D.; Lemon, S.C.; Anderson, B.J.; Stein, M.D. Predictors of follow-up health status in the Drug Abuse Treatment Outcome Study (DATOS). Drug Alcohol Depend. 2003, 69, 243-251. 
7. Simpson, D.; Sells, S. Effectiveness of treatment for drug abuse: an overwiew of the DARP research program. Adv. Alcohol Subst. Abuse 1982, 2, 7-29.

8. Chatham, L.R.; Hiller, M.L.; Rowan-Szal, G.A.; Joe, G.W.; Simpson, D.D. Gender differences at admission and follow-up in a sample of methadone maintenance clients. Subst. Use Misuse 1999, 34, 1137-1165.

9. Gossop, M.; Stewart, D.; Browne, N.; Marsden, J. Methadone treatment for opiate dependent patients in general practice and specialist clinic settings: Outcomes at 2-year follow-up. J. Subst. Abuse Treat. 2003, 24, 313-321.

10. Peles, E.; Schreiber, S.; Adelson, M. Factors predicting retention in treatment: 10-year experience of a methadone maintenance treatment (MMT) clinic in Israel. Drug Alcohol Depend. 2006, 82, 211-217.

11. Gelkopf, M.; Weizman, T.; Melamed, Y.; Adelson, M.; Bleich, A. Does psychiatric comorbidity affect drug abuse treatment outcome? A prospective assessment of drug abuse, treatment tenure and infectious diseases in an Israeli methadone maintenance clinic. Isr. J. Psychiatry Relat. Sci. 2006, 43, 126-136.

12. Teesson, M.; Ross, J.; Darke, S.; Lynskey, M.; Ali, R.; Ritter, A.; Cooke, R. One year outcomes for heroin dependence: findings from the Australian Treatment Outcome Study (ATOS). Drug Alcohol Depend. 2006, 83, 174-180.

13. Cacciola, J.S.; Alterman, A.I.; Rutherford, M.J.; McKay, J.R.; Mulvaney, F.D. The relationship of psychiatric comorbidity to treatment outcomes in methadone maintained patients. Drug Alcohol Depend. 2001, 61, 271-280.

14. Farre, M.; Mas, A.; Torrens, M.; Moreno, V.; Cami, J. Retention rate and illicit opioid use during methadone maintenance interventions: a meta-analysis. Drug Alcohol Depend. 2002, 65, 283-290.

15. Broome, K.M.; Flynnn, P.M.; Simpson, D.D. Psychiatric comorbidity measures as predictors of retention in drug abuse treatment programs. Health Serv. Res. 1999, 34, 791-806.

16. Morral, A.R.; Belding, M.A.; Iguchi, M.Y. Identifying methadone maintenance clients at risk for poor treatment response: pretreatment and early progress indicators. Drug Alcohol Depend. 1999, 55, 25-33.

17. Torrens, M.; Castillo, C.; Perez-Sola, V. Retention in a low-threshold methadone maintenance program. Drug Alcohol Depend. 1996, 41, 55-59.

18. Verthein, U.; Degkwitz, P.; Haasen, C.; Krausz, M. Significance of comorbidity for the long-term course of opiate dependence. Eur. Addict. Res. 2005, 11, 15-21.

19. Schuckit, M.A. Comorbidity between substance use disorders and psychiatric conditions. Addiction 2006, 101, 76-88.

20. Nunes, E.V.; Rounsaville, B.J. Comorbidity of substance use with depression and other mental disorders: from Diagnostic and Statistical Manual of Mental Disorders, fourth edition (DSM-IV) to DSM-V. Addiction 2006, 101, 89-96.

21. Astals, M.; Domingo-Salvany, A.; Castillo-Buenaventura, C.; Tato, J.; Vazquez, J.M.; MartinSantos, R.; Torrens, M. Impact of substance dependence and dual diagnosis on the quality of life of heroin users seeking treatment. Subst. Use Misuse 2008, 43, 612-632. 
22. Torrens, M.; Serrano, D.; Astals, M.; Perez-Dominguez, G.; Martin-Santos, R. Diagnosing comorbid psychiatric disorders in substance abusers: validity of the Spanish versions of the Psychiatric Research Interview for Substance and Mental Disorders and the Structured Clinical Interview for DSM-IV. Am. J. Psychiatry 2004, 161, 1231-1237.

23. Hasin, D.; Samet, S.; Nunes, E.; Meydan, J.; Matseoane, K.; Waxman, R. Diagnosis of comorbid psychiatric disorders in substance users assessed with the Psychiatric Research Interview for Substance and Mental Disorders for DSM-IV. Am. J. Psychiatry 2006, 163, 689-696.

24. Grant, B.F.; Goldstein, R.B.; Chou, S.P.; Huang, B.; Stinson, F.S.; Dawson, D.A.; Saha, T.D.; Smith, S.M.; Pulay, A.J.; Pickering, R.P.; Ruan, W.J.; Compton, W.M. Sociodemographic and psychopathologic predictors of first incidence of DSM-IV substance use, mood and anxiety disorders: results from the Wave 2 National Epidemiologic Survey on Alcohol and Related Conditions. Mol. Psychiatry 2008.

25. Verheul, R. Co-morbidity of personality disorders in individuals with substance use disorders. Eur. Psychiatry 2001, 16, 274-282.

26. Darke, S.; Wodak, A.; Hall, W.; Heather, N.; Ward, J. Prevalence and predictors of psychopathology among opioid users. Br. J. Addict. 1992, 87, 771-776.

27. Pitre, U.; Dansereau, D.F.; Joe, G.W. Client education levels and the effectiveness of node-link maps. J. Addict. Dis. 1996, 15, 27-44.

28. Szapocznik, J.; Ladner, R. Factors related to successful retention in methadone maintenance: a review. Int. J. Addict. 1977, 12, 1067-1085.

(C) 2009 by the authors; licensee Molecular Diversity Preservation International, Basel, Switzerland. This article is an open-access article distributed under the terms and conditions of the Creative Commons Attribution license (http://creativecommons.org/licenses/by/3.0/). 